\title{
MÉTODO DE EJECUCIÓN Y CRITERIO INTERNO DE LA VERDAD EN LA FENOMENOLOGÍA DEL ESPÍRITU
}

\author{
METHOD OF CARRYING IT OUT AND INTERNAL CRITERION OF TRUTH IN THE \\ PHENOMENOLOGY OF SPIRIT
}

\author{
Esteban Sepúlveda ${ }^{1}$ \\ Universidad de Chile
}

Recibido: 19.08.2019 - Aceptado: 01.01.2020

\begin{abstract}
RESUMEN
El presente trabajo aborda lo que Hegel denomina 'método de ejecución' en la Introducción de la Fenomenología del Espíritu. Lo que me propongo es dar una explicación acerca de cómo opera este método tal que logre cumplir las metas que Hegel mismo se propone. En particular, pretendo explicar por qué el objeto para cada figura de la conciencia es cada vez uno distinto y por qué el nuevo objeto surge a partir de la corrección del saber que se encarna en una cierta figura de la conciencia. Me centro en criticar las respuestas a estas preguntas que extraigo de varios autores, con especial atención en cuatro de ellos: Kenneth Westphal (1998), Robert Brandom (2019), Stephen Houlgate (2013) y Robert Pippin (1989). Luego, propongo un bosquejo de mi propia explicación, la cual recurre al uso de un criterio interno de la verdad análogo al que Spinoza emplea para su filosofía.
\end{abstract}

Palabras clave: Método de Ejecución; Experiencia; Criterio Interno de la Verdad; Hegel; Spinoza.

\section{ABSTRACT}

The present article is concerned about what Hegel calls 'method of carrying it out' in the Introduction of the Phenomenology of Spirit. My aim is to give an explanation about how this method works in such a way that it achieves the goals that Hegel impose to himself. In particular, my purpose is to give an explanation about why the new object for each figure of consciousness is in each case a different one, and why the new object emerges from the correction of the knowledge embodied in a particular figure of consciousness. I focus my efforts in criticising the answers to those questions that I got from various authors, whith special attention to: Kenneth Westphal (1998), Robert Brandom (2019), Stephen Houlgate (2013), and Robert Pippin (1989). After I criticise those answers, I will propose a sketch of my own explanation, which resorts to an internal criterion of truth analogous to the one which Spinoza uses in his philosophy.

Keywords: Method of Carrying it out; Experience; Internal Criterion of Truth; Hegel; Spinoza.

${ }^{1}$ Correo electrónico: esteban.scaro@gmail.com. Estudiante de Magíster en Filosofía de la Universidad de Chile. Estudios financiados por la fundación Volcán Calbuco (2020) y por ANID (ex CONICYT): CONICYT-PFCHA/Magister Nacional/2020-22201056. 


\section{INTRODUCCIÓN}

La Fenomenología del Espíritu (PhG) es, sin duda, una obra que ha generado, desde su publicación, una gran cantidad de literatura en torno a ella. Si bien gran parte de la literatura se dirige a pasajes específicos de la $P h G$, existen también trabajos acerca de la unidad sistemática de la $P h G^{2}$ y a su método. Ambos tópicos presentan diversas interpretaciones y difícilmente podría decirse que hay consenso en los puntos en discusión, lo cual muestra la gran dificultad que presenta el texto de Hegel para los intérpretes. En efecto, reina aún la oscuridad en torno a cómo Hegel se propuso cumplir con los objetivos que él mismo le asigna a la ciencia de la experiencia de la conciencia en la Introducción de la $P h G$, a saber, que la meta (el saber absoluto) es una meta necesaria y que el proceso también es él mismo necesario. Esto empeora si consideramos también lo que Hegel dice en la Ciencia de la Lógica (WL, 42/198):

En la Fenomenología del Espíritu he presentado la exposición de la conciencia en su movimiento progresivo: desde la primera, inmediata oposición entre ella y el objeto, hasta el saber absoluto. Ese camino pasa a través de todas las formas de relación de la conciencia con el Objeto, y tiene por resultado el concepto de ciencia. (Énfasis mío.)

Hegel pretende alcanzar estos tres objetivos mediante las consideraciones metódicas que esboza en la Introducción a la PhG. Tenemos, por un lado, que el tránsito necesario de un saber a otro será posible por una negación determinada del saber corregido. Por otro lado, tenemos que el examen del saber obtiene su pauta a partir de la misma conciencia que examina su saber, $y$, en esa medida, la pauta es inmanente a cada figura de la conciencia. La pauta es el lado de la verdad o el en-sí del objeto, mientras que el lado del saber es el para otro del objeto. El examen, entonces, consiste en la comparación de estos dos lados.

$\mathrm{Si}$, al hacer esta comparación, ambas cosas no se corresponden, parece que la conciencia tiene que alterar su saber para hacerlo adecuado al objeto, pero, de hecho, al alterar el saber, se le altera a la conciencia también el objeto mismo; pues

2 El ejemplo paradigmático de esto es Pöggeler 1973. 
el saber que hay es, esencialmente, un saber acerca del objeto; con el saber, también el objeto deviene otro, pues él pertenecía esencialmente al saber. $(P h G 85)^{3}$

Mediante este procedimiento, el examen pasa por todas las figuras de la conciencia hasta llegar al saber absoluto.

Este breve bosquejo de los elementos metódicos de la $P h G$-que, por supuesto, es incompleto- resulta suficiente ya para oscurecer la comprensión del texto de Hegel. Lo que me propongo en este trabajo es tratar de explicar en qué medida es necesario que la $P h G$ opere según la frase recién citada para alcanzar los objetivos que Hegel le asigna. En particular, pretendo explicar por qué el objeto es cada vez uno distinto para cada figura de la conciencia y por qué el nuevo objeto surge del saber corregido. Pues, no hay que ser muy agudo para preguntarse: ipor qué, al detectar la no correspondencia entre el saber y el objeto, y por tanto al modificar el saber para hacerlo adecuado a ese objeto, tendría que surgir un objeto distinto a partir de tal adecuación? La dificultad aumenta si uno considera también este pasaje: "sólo esta necesidad misma, o la emergencia del nuevo objeto que se presenta a la conciencia sin que ésta sepa lo que le acontece, es lo que sucede para nosotros, por así decirlo, como a sus espaldas" (PhG 87). Si esto es así, entonces no es posible apelar a lo declarado (o reflexionado) como en sí por la conciencia de una pretensión de saber para responder estas preguntas, pues el surgimiento del nuevo objeto -que ocurre en correspondencia con el cambio en el saber- no es algo explícito para la conciencia. ${ }^{4}$

Este tópico ha sido pasado por alto por varios comentadores. ${ }^{5}$ No obstante, cuando hay interpretaciones al respecto, estas llegan a variar radicalmente unas de otras. El presente texto se dividirá, a continuación, en seis secciones. En la primera, criticaré brevemente las interpretaciones que son menos sofisticadas. En la segunda, tercera, cuarta y quinta examinaré con más detención las propuestas de Kenneth Westphal (1998), Robert Brandom (2019), Stephen Houlgate (2013) y Robert Pippin (1989),

${ }^{3}$ Citaré la $P h G$ señalando el párrafo al cual pertenece el pasaje citado; los párrafos son numerados de manera consecutiva.

${ }^{4}$ Por esto rechazo la explicación que Kenley Dove 1998, 57 da al respecto. También Hans-Friedrich Fulda 2008, 31 afirma que la aparición de este nuevo objeto también es para la conciencia examinada; sin embargo, él no da mayores razones para sostener su lectura.

5 Ejemplos de ello son: Eugen Fink 2011; Ramón Valls Plana 1994; Jean-Pierre Labarrièrre 1985; Frederick Beiser 2005. En ellos hay poco o nada relevante escrito sobre este tema. Terry Pinkard 1994, si bien da una interpretación de en qué medida el tránsito al nuevo saber es necesario, omite por completo en qué medida sería necesario también el tránsito a un nuevo objeto; lo mismo ocurre con Robert Stern 2002. 
respectivamente. Finalmente, expondré un bosquejo de lo que, a mi parecer, logra explicar estos puntos.

\section{EXPLICACIONES DE ESTA NECESIDAD}

En primer lugar, criticaré una tendencia interpretativa que denominaré 'interpretación por eliminación'. La interpretación por eliminación considera que el tránsito de figuras consiste en la refutación de todas las pretensiones de saber que no corresponden a la que Hegel concibe como el saber absoluto. Dentro de esta posición ubico a autores tales como Ludwig Siep, Kenneth Westphal e Ioannis Trisokkas ya sea por afirmarlas expresamente, ya sea por falta de ulterior explicación. En efecto, Trisokkas afirma que

La estrategia de Hegel contra el Pirronista es mostrar (a) que el proponer este criterio general no sucumbe ante el argumento del criterio de la verdad y (b) que todas las figuras de la conciencia fallan al satisfacer ese criterio. (Trisokkas 2012, 78)

Siep, por su parte, sostiene que

Este punto de vista [el de la ciencia] tiene que justificarse ante la conciencia natural y ofrecerle a ella una 'escala' mediante el examen inmanente y la refutación de todas las formas del saber, todos los órdenes del obrar, todas las figuras de la cultura que se hallan marcadas por esta posición o a las que les es inherente esta convicción de una oposición de objeto y sí mismo. La Introducción expone el método de este examen. (Siep 2015, 66)

Finalmente, Westphal afirma que

Un punto central a las consideraciones de Hegel en torno a la 'negación determinada' es su tesis de que una teoría del conocimiento (como cualquier otra teoría filosófica) solo puede ser justificada mediante una crítica exhaustiva y estrictamente interna de las teorías del conocimiento alternativas. (Westphal 2003, 46)

Si bien considero que esta interpretación, en cierto aspecto, es correcta (a saber, en el hecho de que Hegel se propone mostrar la nulidad de un aspecto de toda pretensión de saber que no sea la que corresponda al saber absoluto), es completamente insuficiente como interpretación del modo en que Hegel pretende alcanzar los objetivos que le asignó a la $P h G$; y, por consiguiente, es una interpretación insuficiente del método de ejecución. En efecto, esta interpretación alcanza tan solo a dar cuenta de la necesidad de la meta, pero de ningún modo de la necesidad del proceso, a saber, del por qué una cierta figura de la conciencia ha de suceder necesariamente a otra. Tanto la necesidad de la meta como la del proceso son condiciones que, según Hegel, el método de ejecución debiera lograr satisfacer: "la meta le está 
fijada al saber tan necesariamente como la serie del proceso" (PhG 80). Además, si no da cuenta de la necesidad del proceso, entonces tampoco puede dar cuenta de otro aspecto de la relación entre las figuras de la conciencia: la relación de conservación que existe entre las figuras de la conciencia (la superación ${ }^{6}$ de una figura por otra).

A continuación, criticaré la explicación que ofrece H.S. Harris sobre el surgimiento del nuevo objeto. Harris ve en la experiencia un proceso de aprendizaje de la conciencia, tal que "la experiencia cambia el hecho que se conoce de ser un simple hecho a ser un hecho conocido. La verdad no es más que p es de tal modo, sino que es sabido que p es de tal modo" (Harris 1997, 187). En consecuencia, "mi conocimiento del hecho cambia el objeto del 'conocimiento', pues es necesariamente el caso que la totalidad de mi conocimiento no es simplemente el hecho de que es de día, sino el hecho de que yo sé que es de día" (Harris 1997, 186). Este mismo proceso se aplicaría al estándar de la verdad (a la pauta). Lo que se busca es saber en qué consiste conocer. Cada experiencia mostraría un hecho acerca del conocer que sería, de esta manera, incorporado al saber de la conciencia, y así hasta llegar al saber absoluto.

El problema de la propuesta de Harris es que su explicación del surgimiento del nuevo objeto no es lo suficientemente clara. Pues falta todavía explicar el gran paso que va desde considerar que el objeto cambia de ser un mero hecho a ser un hecho conocido - por ejemplo, de que el carácter mediado del espacio y el tiempo (o de cualquier referencia a singulares) pase de ser un mero hecho a ser un hecho conocido - hasta considerar que el objeto refleja este saber de él como naturaleza - por ejemplo, que los mismos singulares tengan en ellos mismos (no en la referencia a éstos) este mismo carácter mediado, tal que sea una cosa con muchas propiedades. En esta transición hay un salto que la explicación de Harris no contempla.

Finalmente, criticaré la explicación que ofrece Walter Jaeschke. Este autor intenta explicar la necesidad en el surgimiento del nuevo objeto del siguiente modo:

la conciencia a la que le desaparece su verdad no puede quedarse sin verdad: esto constituye su necesidad estructural. De ella, además, no puede sustraerse por sí misma una conciencia que rinda homenaje a un escepticismo dogmático y forzado, puesto que una conciencia sin verdad sería una conciencia sin objeto y por ello más bien no sería conciencia alguna. (Jaeschke 2010, 47)

${ }^{6}$ Véase el comienzo de los capítulos IV y VI de la $P h G$. 
Sin embargo, la necesidad de que la conciencia tenga algo verdadero (esto es, que tenga un objeto para ella concebido como lo en sí) no alcanza a explicar por qué una concepción de objeto en concreto sucede necesariamente a otra, pues lo único que es necesario, según la interpretación de Jaeschke, es que haya un objeto (sin determinar necesariamente cuál sea este).

\section{LA PROPUESTA DE KENNETH WESTPHAL}

Kenneth Westphal (1998) también desarrolla una concepción más detallada sobre el método de ejecución. La propuesta de Westphal consiste en distinguir entre 8 elementos que entrarían en juego en la experiencia. 1) La concepción que la conciencia tiene del mundo o el mundo según la conciencia (the world according to consciousness): este elemento corresponde al en sí que la conciencia declara y que conforma el criterio mediante el cual la conciencia se examina a sí misma. 2) el mundo tomado como una instanciación de la concepción que la conciencia tiene del mundo o el mundo para la conciencia (the world for consciousness): este elemento es descrito por Westphal de la siguiente manera: "un objeto es para la conciencia cuando la conciencia aplica su concepción de objeto al objeto mismo, cualquiera sea éste" (Westphal 1998, 88), donde la concepción de objeto aplicada corresponde a 1). 3) Aquellos elementos del mundo que están relacionados con -pero no incluidos en (son implícitos a)- la concepción que la conciencia tiene del mundo o el mundo a los ojos de la conciencia (the world to consciousness): estos elementos corresponden a aquello que la conciencia descubre del objeto una vez hecha la experiencia. 4) El mundo tal cual es, con todas sus propiedades, ya sea conocidas o desconocidas, o el mundo mismo (the world itself). Esta misma estructura se repite con respecto al conocimiento, enumerada con letras en vez de números ( 1 corresponde a $\mathrm{A}, 2$ corresponde a $\mathrm{B}$, etc.).

Siguiendo el esquema de Westphal, entonces, el examen que la conciencia hace de sí consiste en ver si su concepción del mundo y del conocimiento ( 1 y A) corresponden con el mundo y el conocimiento tal cual son para la conciencia ( 2 y B). La objetividad de este examen está garantizada porque 2 y B son el resultado de la aplicación de 1 y A en el mundo y el conocimiento tal cual son ( 4 y D). Si el resultado es que 1 y A no corresponden a 2 y $\mathrm{B}$, entonces la conciencia se torna transparente de nuevos elementos que ha de tener en consideración en la concepción que ella posee del mundo y del conocimiento (3 y C). Así, 
Mediante una aplicación escrupulosa y exhaustiva de principios epistémicos y ontológicos, aquellos rasgos de los objetos que no han sido considerados por tales principios pueden hacerse explícitos. Tales descubrimientos pueden requerir solo reconsiderar la importancia de los rasgos previamente reconocidos, aunque dejados sin considerar, de los objetos; o pueden implicar el reconocer rasgos previamente desconocidos del conocimiento o del mundo (...) Al hacer manifiestos de este modo los rasgos del conocimiento o del mundo previamente desconocidos o dejados sin considerar, las expectativas refutadas proveen información que puede ser usada para revisar las concepciones del mundo y del conocimiento. (Westphal 1989, 92)

De este modo, la conciencia puede seguir examinando si su concepción del mundo y del conocimiento corresponden a como el mundo y el conocimiento son para ella.

Sin considerar las discutibles distinciones que Westphal introduce, ${ }^{7}$ las observaciones críticas que me interesa exponer aquí son dos. En primer lugar, lo que se modifica en la experiencia es nuestro saber, en la medida en que este se adecúa al objeto, y es a partir de esta modificación como surge el nuevo objeto:

$\mathrm{Si}$, al hacer esta comparación, ambas cosas no se corresponden, parece que la conciencia tiene que alterar su saber para hacerlo adecuado al objeto, pero, de hecho, al alterar el saber, se le altera a la conciencia también el objeto mismo; pues el saber que hay es, esencialmente, un saber acerca del objeto; con el saber, también el objeto deviene otro, pues él pertenecía esencialmente al saber. (PhG 85)

Sin embargo, Westphal no da cuenta de esta relación de dependencia, sino que tan solo asume que la concepción del nuevo objeto se da a partir del reconocimiento de ciertos elementos y no de la modificación del saber (la concepción del conocimiento) de ese objeto. 0 bien, puesto de otro modo, los elementos que la conciencia reconsidera tanto del mundo como del conocimiento no tienen ningún principio que oriente este proceso, de tal modo que la correspondencia que el nuevo objeto guarda con el saber modificado queda sin explicar.

En segundo lugar, la formación del nuevo objeto no es algo que sea patente para la conciencia: "sólo esta necesidad misma, o la emergencia del nuevo objeto que se presenta a la conciencia sin que ésta sepa lo que le acontece, es lo que sucede para nosotros, por así decirlo, como a sus espaldas" ( $P h G$ 87). En consecuencia, no ocurre, tal como lo plantea

7 Es extraño que Westphal hable de la aplicación de una concepción del mundo al mundo tal cual es en realidad, pues ¿en qué consiste esa aplicación? ¿cómo se la realiza? ¿cómo uno puede no errar o engañarse al realizarla? Resulta aún más extraño que de una tal aplicación resulte un saber, como si adquirir un conocimiento del mundo fuera el simple proceso de aplicar una concepción del mundo al mundo mismo. Para más críticas véase la nota 9. 
Westphal, que la conciencia se da cuenta de ciertos elementos del mundo que no consideró antes y que, con esta nueva concepción del mundo, vuelve a examinar su nueva concepción del objeto. De este modo, Westphal yerra al explicar el modo en que Hegel presenta las relaciones entre el objeto y el saber en el tránsito de una figura a otra.

\section{LA PROPUESTA DE ROBERT BRANDOM}

Robert Brandom ofrece la siguiente interpretación de por qué, al modificar el saber, se modifica también el objeto. Él distingue entre lo que en cada caso es el en sí "a los ojos de" [to] ${ }^{8}$ la conciencia y lo que es su objeto. Mientras que "ser un en-sí es ser lo que la conciencia prácticamente toma o trata como real" (Brandom 2019, 91) -que por cierto es implícito para la conciencia-, el objeto es la representación que uno tiene de ese objeto tal como es realmente. De esta manera, por ejemplo, si el juicio de que el palo está doblado es detectado en su error, una vez que yo saco el palo del agua,

El 'nuevo y verdadero objeto' es la representación del palo doblado revelada como errónea, como una representación errada de lo que es ahora, a los ojos [to] del sujeto, el modo en que las cosas son realmente: un palo recto. Esta representación es 'verdadera', no en el sentido de representar cómo las cosas son realmente, sino en el sentido de que ahora es, a los ojos [to] de la conciencia, lo que es realmente el caso: una mera apariencia, una representación errada" (Brandom 2019, 91-92).

Esto le permite explicar a Brandom en qué sentido, al modificar el saber, se modifica también el objeto, pues

Lo que se altera es el estatus de la representación del palo doblado, lo que es a los ojos de [to] la conciencia. Ella ha disfrutado el estatus de ser a los ojos de [to] la conciencia lo que el palo es en sí mismo. Pero ahora su estatus ha cambiado a ser a los ojos de [to] la conciencia solo lo que el palo era para [for] la conciencia: una apariencia. (Brandom 2019, 92)

De este modo, Brandom ofrece una respuesta a las preguntas que aquí persigo. Sin embargo, hay un gran malentendido en el modo en que Brandom analiza el asunto. Pues Brandom $(2019,103)$ considera que el análisis del saber que se examina en una cierta figura de la conciencia ha de ser atingente también cuando el análisis es referido al saber de un objeto particular (el palo doblado, por ejemplo). Así, cuando mi saber se muestra

8 Antonio Gómez Ramos traduce la expresión alemana ihm Bewußtsein (en inglés to consciousness) como 'a los ojos de la conciencia', a fin de distinguirla de la expresión für Bewußtsein (en inglés for consciousness). Entiendo que puede ser extraño para el lector que Brandom considere que algo "a los ojos de" la conciencia sea, sin embargo, implícito para la misma conciencia. Esta dificultad es producida meramente por la traducción elegida. Para una justificación y un mayor tratamiento sobre este punto, véase la nota siguiente. 
errado, Brandom $(2019,89)$ dice: "cuando me doy cuenta de que el palo que yo tomaba por estar doblado está en realidad recto, mi perspectiva acerca del palo cambia, pero el palo mismo no cambia". Ciertamente, adoptar esta perspectiva lo lleva a distinguir entre lo que es en sí a ojos de la conciencia y lo que es el segundo objeto. Sin embargo, si abandonamos esa pretensión de Brandom y consideramos el asunto como es realmente, a saber, que lo que es objeto en el análisis de Hegel no refiere nunca a un objeto particular, sino a la forma de la objetividad, es decir, refiere a aquello -que, ciertamente, posee ciertas características, como ser, por ejemplo, inmediato sensible particular- que vale como objeto para la conciencia, tal distinción resulta innecesaria y sin justificación.

Si uno entiende que esto (la forma de la objetividad) es lo en sí, entonces no hay problema con que el en sí cambie. Pues no es que, al cambiar el en sí, cambie la realidad, es decir, cambie el palo de estar realmente doblado a estar realmente derecho, sino que cambia lo que consideraremos en cada caso como lo real. Por otro lado, la distinción de Brandom tampoco se sostiene en el texto. En efecto, no es difícil encontrar pasajes donde Hegel afirma que el objeto no se distingue del en sí. Por ejemplo, Hegel ( $P h G$ 84) afirma: "lo esencial es retener para toda la investigación que estos dos momentos, el concepto y el objeto, ser para otro y ser en símismo, caen ambos dentro del saber que estamos examinando" (énfasis mío). ${ }^{9}$

${ }^{9}$ Lo único que motivaría aún a Brandom a distinguir entre en sí y ser objeto es la interpretación que él ofrece de la diferencia entre ser to [ihm] consciousness y ser for [für] consciousness. Sin embargo, esta va más allá de lo que el texto mismo permite. En efecto, Brandom -al igual que Westphal 1998, 89- afirma que la distinción hay que entenderla como lo que es implícito [ihm] y lo que es explícito [für]. Sin embargo, el texto es esquivo a admitir esa distinción, al menos en el párrafo que ellos señalan. El pasaje al que remonta la distinción es el siguiente: "Pero justo en el simple hecho de que ella sepa acerca de un objeto viene ya dada la diferencia de que algo sea lo en sí a sus ojos [ihm], mientras que el saber, o el ser objeto para [für] la conciencia, es otro momento" (PhG 85). Sin embargo, este pasaje tiene que ser leído en conjunto con otro del mismo párrafo, pero un poco más arriba: "Pues la conciencia es (...) conciencia de lo que a ella [ihm] le es lo verdadero, y conciencia de su saber acerca de ello. Siendo ambas cosas para [für] ella misma, ella misma es la comparación de ambas" (énfasis mío). Lo que muestra este último pasaje es que, sea cual sea la distinción entre ihm y für, lo que es ihm también es für. Con esto, se cae la distinción entre lo implícito [ihm] y lo explícito [für], pues lo implícito tendría que ser ello mismo también explícito. Es más, para que la conciencia misma sea su comparación, esta tiene que tener explícitos ambos lados de la comparación. En mi opinión, la distinción entre ihm y für debe ser leída en relación con lo que Hegel mismo establece como lo que es en sí y lo que es para otro: "Dentro de ella hay una cosa que es para otra, o bien, a la conciencia como tal le es inherente la determinidad del momento del saber; a la vez, a sus ojos, esta otra cosa no es sólo para ella, sino que está también fuera de esta relación, o es en sí; el momento de la verdad" (PhG 84). Ahora bien, como ambas cosas son para la conciencia, ello fuerza a Hegel a distinguir, dentro de lo que es para [für] la conciencia, aquello que es el saber (nuevamente, para [für] la conciencia) de aquello que es así también fuera del saber de ello (lo que es a ojos de [ihm] la conciencia). 
Por último, aun si se acepta la interpretación de Brandom, esta no ofrece ninguna explicación acerca de en qué medida el surgimiento del nuevo en sí es uno necesario. La postura que él defiende es que la necesidad del proceso radica en la mirada retrospectiva de quien ya ha llegado al saber absoluto.

Del proceso mismo de las experiencias pasadas, el recolector selecciona una trayectoria que es exhibida como expresivamente progresiva -esto es, como teniendo la forma de una revelación gradual y acumulativa de cómo las cosas son realmente (según el recolector) (...). Que en este camino el pasado sea constantemente convertido en una historia (...) es como Hegel comprende la razón como, retrospectivamente, 'dando a la contingencia la forma de la necesidad'. (Brandom 2019, 102)

Esta interpretación hace caso omiso del hecho de que Hegel señala que el proceso es necesario, no solo en el final, sino durante el proceso mismo. En efecto, Hegel afirma que es el mecanismo de tránsito de una figura a otra $-y$, por tanto, de un objeto a otro- el que es responsable de la necesidad del proceso: "es esta circunstancia la que guía toda la serie de figuras de la conciencia en su necesidad" (PhG 87).

\section{LA PROPUESTA DE STEPHEN HOULGATE}

Pasaré ahora a examinar la propuesta de Stephen Houlgate. Este autor propone invertir la relación de dependencia entre el saber y el nuevo objeto; es decir, para él el nuevo objeto no surge a partir de la modificación del saber, sino que el saber es modificado precisamente debido a que el objeto $y a$ ha cambiado. ${ }^{10}$ Houlgate establece su interpretación a partir de una ambigüedad en la referencia de la 'alteración' que sufre el objeto de la conciencia:

Esa palabra, sugiero, refiere no a la alteración que 'pareciera' que la conciencia debería hacer para traer su conocimiento de vuelta a la concordancia con su objeto, sino que refiere a la alteración por medio de la cual el conocimiento viene en primer lugar a diferir de la concepción inicial del objeto (...). Esto sugiere que el objeto se altera, no cuando revierto el verlo como originalmente lo veía, sino en el proceso mismo de ser conocido en primer lugar. (Houlgate 2013,19)

En consecuencia, el argumento de Hegel en torno a la necesidad del cambio del saber y del objeto ha de entenderse de la siguiente manera:

(1) Tomo el objeto como siendo X; (2) llego a conocerlo como siendo Y; (3) de este modo, mi conocimiento no se adecúa al objeto; (4) pareciera, entonces, que

10 También Jean Hyppolite 1974, 25 sostiene esta interpretación: "tal es precisamente el resultado de la experiencia: la negación del objeto precedente y la aparición de un objeto nuevo que, a su vez, origina un nuevo saber". Igualmente, Harris 1997, 187: "el mundo cambia y nuestro conocimiento cambia con él". 
debería alterar mi conocimiento para hacerlo adecuado al objeto, que debería revertir el conocerlo como siendo X; (5) sin embargo, no puedo revertir esa concepción inicial del objeto porque, en la alteración de mi conocimiento que ya ha tenido lugar, el objeto mismo ha sido alterado ante mis ojos: el objeto ha probado no ser simplemente X, sino ser Y; (6) esta alteración del objeto es irreversible, porque el conocimiento que hemos llegado a tener del objeto no es erróneo, sino genuino conocimiento del objeto (Houlgate 2013, 19).

La interpretación de Houlgate tiene serias dificultades textuales. Pues, en efecto, es claro en el texto de Hegel que la alteración del objeto viene dada a causa de la alteración del saber: "al alterar el saber, se le altera a la conciencia también el objeto mismo (...) con el saber, también el objeto deviene otro" (PhG 85). No obstante, las dificultades de su interpretación no son solo textuales. En efecto, por un lado, si uno le preguntara a Houlgate: “¿mirando a qué el saber se modifica?", la respuesta apropiada sería: "mirando al nuevo objeto"; pero esta respuesta no da cuenta del carácter inmanente del examen de cada figura de la conciencia. Pues, Hegel menciona que el surgimiento del nuevo objeto ocurre a espaldas de la conciencia (PhG 87). En consecuencia, el nuevo objeto le aparece a la conciencia como algo encontrado. Si esto es así, entonces ¿qué razón tendría la figura de la conciencia en cuestión para detectar la no correspondencia entre su saber y el objeto? Pues ella podría perfectamente alegar que el examen no cuenta, porque le han cambiado el objeto: si la conciencia no toma nota de que el primer objeto deviene en el segundo, ¿en qué medida la pauta (el objeto) sigue siendo inmanente a la conciencia examinada y a su saber?

Por otro lado, la interpretación de Houlgate no puede dar cuenta de la necesidad del tránsito de una figura a otra. En efecto, esta necesidad está dada por la modificación en el saber. Ahora bien, la modificación del saber, según esta interpretación, resulta de la modificación en el objeto. Sin embargo, si uno se pregunta: “¿atendiendo a qué cambia el objeto?”, no hay una respuesta clara en esta interpretación. ¿Por qué el objeto de la Certeza Sensible resulta ser en realidad el objeto de la Percepción y no el objeto de Fuerza y Entendimiento, incluso el objeto del deseo? Todas las consideraciones metódicas de Hegel en la Introducción están dirigidas a entender la necesidad del tránsito de un saber a otro. Por consiguiente, parece ser que la interpretación de Houlgate no puede explicar por qué el objeto de la Certeza Sensible necesariamente resulta ser el objeto de la Percepción, tal que la Certeza Sensible modifique necesariamente su saber para hacerlo adecuado al saber de Percepción. 


\section{LA PROPUESTA DE ROBERT PIPPIN}

Pippin no afirma explícitamente la interpretación que le adjudico; sin embargo, esta puede extraerse de su libro Hegel's Idealism (1989). Para facilitar la referencia a esta interpretación, me referiré a ella como 'la interpretación de Pippin'. Este autor considera que cada figura de la conciencia refleja una concepción sobre cómo es posible la experiencia de objetos:

Hegel está presuponiendo que hay varias maneras fundamentales en las cuales quien tiene experiencia puede entender su relación con los objetos, que tales Conceptos [Notions], o presuposiciones acerca de lo Absoluto, son condición para la posibilidad de la experiencia, al menos en el sentido de que ellas no son revisables de cara a la experiencia (Pippin 1989, 102-103).

Si esto es así, el examen de la conciencia consistiría en lo siguiente:

Lo que está en cuestión para Hegel es si el 'Concepto de sí' de la conciencia, su presuposición tanto de lo que hay realmente para conocer (...) como del modo en el cual ella, la conciencia, puede conocer tal realidad (...) son, en un momento o en otro, tal como ella las toma. Su interés está en si una cierta concepción de la experiencia, una autocomprensión acerca de las condiciones bajo las cuales una relación judicativa con los objetos puede ser establecida, puede de hecho ser exitosa en dar cuenta de tal relación judicativa (Pippin 1989, 106).

La interpretación de Pippin ofrece una respuesta a las preguntas que persigo en este escrito. Pues, el objeto es cada vez uno distinto y cambia debido al cambio en el saber, porque el objeto no es nada más que lo que el saber, en cada caso, proponga que sea; y el saber, a su vez, no es nada más que una concepción de qué es el objeto y cómo se lo conoce. Ahora bien, detectar la inadecuación entre el saber y el objeto solo es posible, para Pippin, a partir de otro saber, a partir de otra concepción o Concepto de la experiencia. "Tal Concepto [Notion] es necesario para que haya experiencia; hay experiencia y, por ende, la pregunta por la legitimidad solo puede surgir relativa a otros Conceptos [Notions] posibles" (Pippin 1989, 107). En este sentido, cada vez que cambia el saber, forzosamente cambia el Concepto, es decir, la concepción de cómo es posible la experiencia de objetos; pues el cambio en el saber se debe a la detección de la inadecuación entre el saber y el objeto, inadecuación que ella misma solo es posible de captar desde otro Concepto. El nuevo saber es el que correspondería al que asume este último Concepto. Asimismo, cada vez que cambia el saber, forzosamente cambia el objeto, pues el objeto no es nada más que lo que el Concepto dicta que él es, $y$, cada vez que cambia el saber, cambia el Concepto.

Mi desacuerdo con esta interpretación brota de la diferencia entre nuestras lecturas de los primeros párrafos de la Introducción de la $P h G$. 
Pippin sostiene que la $P h G$ está, completamente y desde el inicio, orientada a la solución de lo que él denomina 'escepticismo trascendental'.

La cuestión que diferencia radicalmente a Hegel de Kant y Fichte es expresada en la acusación de que Kant y Fichte, a pesar de sus logros, son escépticos, filósofos que finalmente restringieron sus propios resultados al admitir que no tienen ningún modo de establecer que las condiciones para una experiencia de objetos posiblemente autoconsciente son genuinamente objetivas. (Pippin 1989, 92)

Para Pippin, la $P h G$ es la deducción del concepto de ciencia ( $W L$, 43/198), en la medida en que esta es el procedimiento mediante el cual la anticipación categorial en la experiencia de objetos queda legitimada en su uso. Además, tal legitimación se logra, por un lado, sin apelar a la intuición pura kantiana; por otro, evitando caer en el escepticismo trascendental recién mencionado.

Siguiendo esta idea, Pippin considera que el saber, que se critica en los primeros párrafos de la Introducción y frente a los cuales la ciencia sería, primeramente, tan solo una aparición, es todo aquel saber que asuma el giro crítico kantiano. ${ }^{11}$ Es decir, la extensión de la referencia al "saber que aparece" remite tan solo a lo que cae bajo el rótulo de "filosofía trascendental". Pippin $(1989,95)$ niega rotundamente que Hegel esté interesado en otro tipo de escepticismo: "Al igual que Kant, Hegel está, desde el inicio, interesado en las condiciones de posibilidad del conocimiento, y en ningún lugar parece estar interesado en el problema moderno postcartesiano: ¿hay siquiera alguna buena razón para creer que nosotros conocemos algo?". De este modo, a partir de lo que Pippin sostiene, se sigue que cada figura de la conciencia debe considerarse como una propuesta de filosofía trascendental, una concepción acerca de cómo es posible la experiencia de objetos.

Discrepo en dos puntos con Pippin. El primero de ellos es respecto a la extensión de la referencia del "saber que aparece". Si bien es cierto que en el primer párrafo de la Introducción Hegel parece tener en mente a Kant, ${ }^{12}$ el texto es lo suficientemente ambiguo como para pretender referirse no solo a Kant, sino a cualquier empresa cognitiva que opere bajo la "representación natural" en filosofía. No solo eso, sino incluso lo dicho en el párrafo 5 de la Introducción podría entenderse perfectamente contra lo que Pippin sostiene. Pues ahí Hegel (PhG 76) afirma que

11 Robert Stern 2002, 39 asume esta postura explícitamente.

12 En la sección decicada a Kant de las Lecciones sobre la Historia de la Filosofía III (VGPh III, 333-334/421), aparecen las mismas descripciones. 
En lugar de andar torturándose sin provecho con semejantes representaciones y modos de hablar acerca del conocimiento como un instrumento para hacerse con lo absoluto (...) las representaciones podrían desecharse sin más, por contingentes y arbitrarias; y el uso que, ligado a ellas se hace de palabras como lo absoluto, el conocer, lo objetivo y lo subjetivo, y muchísimas otras, cuyo significado se da por consabido, podría considerarse incluso como un fraude.

En este pasaje, si se asume la lectura de Pippin, Hegel estaría sugiriendo que hay que abandonar, como punto de partida para determinar en qué consiste la ciencia (el saber absoluto), la filosofía trascendental de corte kantiano.

Asimismo, el que "la ciencia, por el hecho de entrar en escena, es ella misma una aparición" (PhG 76) y lo que sigue a esa afirmación, es esbozado de un modo tan general y vago que difícilmente podríamos asumir -sin ulterior argumentación- que ahí Hegel solo tiene en mente las variaciones de la filosofía trascendental. Es más, hay incluso evidencia para pensar lo contrario. Tal como lo afirma Forster, la pretensión de Hegel en el periodo de Jena era la de proveer una justificación de la ciencia para el punto de vista no filosófico: "Al final del periodo de Jena, en la Fenomenología, Hegel emprendió su más temprano y explícito intento de satisfacer este estándar epistemológico adicional de proveer una prueba de la Ciencia Filosófica para todos los puntos de vista no científicos" (Forster 1989, 114), lo cual se ve reforzado con la siguiente afirmación de Hegel en el Prólogo: "el individuo tiene el derecho a exigir que la ciencia le alcance la escalera para llegar, cuando menos, hasta ese punto de vista" (PhG 26). Si esto es así, entonces Hegel no pudo tener a la vista en la Introducción solo filosofías de corte trascendental.

Si se admite lo anterior, entonces, en el contexto del examen del saber de la conciencia, se debe procurar no tener como supuesto del examen a la ciencia ya desplegada en su verdad. En otras palabras, para que sea posible desplegar la ciencia de la experiencia de la conciencia, Hegel debe esclarecer los supuestos básicos acerca del conocimiento a partir de los cuales el examen puede tener cabida. Ahora bien, tales supuestos básicos son, a fin de cuentas, supuestos que la ciencia (el saber absoluto), de algún modo, también tiene que incorporar. La pregunta fundamental, entonces, es la siguiente: ¿cuánto de la ciencia se puede suponer para que el examen tenga cabida sin que ello nos diga ya qué aparición del saber no corresponde a la ciencia? La interpretación de Pippin admitiría demasiado como punto de partida del examen del saber de la conciencia. Pues, aun cuando sea verdadero que, para Hegel, tal como Pippin lo afirma, a cada figura de la conciencia subyace un Concepto [Notion] que articula la experiencia y el saber de tal figura de la conciencia, si ello se admite desde el inicio, entonces 
ya se habrá determinado qué apariciones del saber no corresponden a la ciencia (i.e. toda la que no sea filosofía trascendental).

En relación con esto último, el segundo punto de mi desacuerdo con Pippin es que de su interpretación se sigue que hay que leer las tres primeras secciones en tanto que apelarían a condiciones de posibilidad de la experiencia. Sin embargo, cuesta trabajo entender en qué medida apelar a una cosa que subyace a sus múltiples propiedades, o apelar al juego de fuerzas, fueran apelaciones a condiciones de posibilidad de la experiencia, si ninguna de ellas es, en rigor, experimentable por la conciencia; y, no obstante, se apela a ellas como si tuvieran realidad objetiva. De hecho, si uno sigue el hilo de la $P h G$, lo razonable sería pensar que, una vez que se llega a la perspectiva de la Autoconciencia, se determinaría que lo que sea la ciencia, lo será como una propuesta de filosofía trascendental. A causa de estos dos puntos débiles, rechazo la propuesta que extraigo del libro de Pippin.

\section{MI PROPUESTA: UN RECURSO A SPINOZA}

La explicación que yo propongo, de por qué Hegel considera que el objeto ha de ser cada vez uno distinto y de por qué el nuevo objeto surge a partir de la alteración del saber, es que el examen opera apelando a un criterio interno de la verdad. El empleo de este criterio interno de la verdad sería similar al que Spinoza haría de él en su filosofía. La tesis de Spinoza es básicamente la siguiente: es posible determinar la verdad de una idea no solo atendiendo a si la idea corresponde con lo ideado por ella, sino también atendiendo a la manera en que es formulada tal idea. Dicha manera corresponde al criterio interno de la verdad, y tal criterio interno de la verdad es, en la filosofía de Spinoza, lo que él denomina idea adecuada. Así, Spinoza define en Ética II, d. 4 (EIId4) la idea adecuada del siguiente modo: "por idea adecuada entiendo la idea que, en cuanto que se considera en sí misma, sin relación al objeto, tiene todas las propiedades o denominaciones intrínsecas de la idea verdadera"; y en la explicación de esta definición, aclara: "digo intrínsecas a fin de excluir aquella que es extrínseca, a saber, la concordancia de la idea con su objeto ideado".13

13 Véase también la carta 60: "Entre la idea verdadera y la adecuada no reconozco otra diferencia, sino que el término verdadero sólo se refiere a la conveniencia de la idea con su objeto (ideatum), mientras que el término adecuado se refiere a la naturaleza de la idea en sí misma; así que no se da ninguna diferencia entre la idea verdadera y la adecuada fuera de aquella relación extrínseca". 
De esta perspectiva se sigue fácilmente lo que Spinoza afirma en EIIp34, a saber, que "toda idea, que en nosotros es absoluta o adecuada y perfecta, es verdadera". Por último, si una idea es determinada como adecuada, y por tanto como verdadera en virtud de su denominación intrínseca, asegura por ello su concordancia con lo ideado por esa idea. El que esta concordancia esté asegurada por la determinación de una idea como adecuada, está garantizado por lo siguiente, a saber, que, por un lado, "todas las ideas que se siguen en el alma de ideas que en ella son adecuadas, son también adecuadas" (EIIp40) y, por otro, que "el orden y la conexión de las ideas es el mismo que el orden y la conexión de las cosas" (EIIp8). De este modo, el orden y la conexión de ideas adecuadas le permiten a Spinoza anticipar el orden y la conexión del mundo.

Spinoza llega a la tesis de que hay un criterio interno de la verdad en el Tratado de la Reforma del Entendimiento (TIE) de un modo bastante similar al que utiliza Hegel en su Introducción de la PhG. Al igual que Hegel,14 la reflexión de Spinoza inicia con su rechazo a determinar cuándo uno está en posesión del saber antes de saber algo. Esto lo muestra desde dos perspectivas. Por un lado, si consideramos que antes de investigar hay que definir el método correcto de investigación, esto último también requerirá de un método, y así hasta el infinito (TIE 30); o bien, por otro lado, si la idea verdadera es algo distinto de su correlato, entonces es algo real y por ello algo inteligible, es decir, su esencia formal está contenida objetivamente en una nueva idea, la cual, a su vez, es distinta de su correlato, lo cual también nos lleva a una regresión al infinito (TIE 33). La propuesta de Spinoza es considerar el método como conocimiento reflexivo. El método, rectamente entendido, no es nada más que el conocimiento que podemos obtener de una idea que ya consideramos verdadera, para, a partir de ahí, comprender cuál es la forma de la verdad y formar el resto de nuestras percepciones con aquella forma como norma, y también distinguir la percepción verdadera del resto. Todo lo que se requiere es una idea verdadera dada.

Mi propuesta es que Hegel también adscribe a esta concepción del método, y que, en consecuencia, la $P h G$ tiene que verse como el constante intento y fracaso -cada vez con mayor sofisticación- de ejecutar este método. En este sentido, Hegel determinaría para cada figura cuál es su

${ }^{14}$ Estas son las primeras líneas de la Introducción: "la representación natural es que, en filosofía, antes de ir a la Cosa misma, esto es, al conocimiento efectivo de lo que es en verdad, sería necesario entenderse previamente sobre el conocimiento (...). Esta preocupación tendrá incluso que transformarse en la convicción de que toda la empresa de que la conciencia, por medio del conocimiento, llegue a adquirir aquello que es en-sí, debe de ser un contrasentido en su mismo concepto, y que entre el conocimiento y lo absoluto se alza una frontera que, simple y llanamente, los separa" (PhG 73). 
criterio interno de la verdad, y lo que se examinaría es la aptitud de ese criterio como criterio interno de la verdad en la correspondencia entre el saber que aparece y el objeto, de modo que, una vez explícita la no correspondencia entre ambos, se avanza a otro saber (y, por tanto, a otro criterio interno de la verdad), y así hasta llegar al saber absoluto.

Existe también una similitud entre lo que Spinoza entiende en concreto por idea adecuada y la manera en que Hegel entiende el saber que aparece. La idea adecuada consiste en el conocimiento por causa próxima (en el caso de los entes creados), o por su propia esencia (en el caso del ente increado). Por causa próxima se entienden aquellos axiomas y nociones comunes que conforman la esencia de algo, esto es, que presentan los componentes de la génesis de algo. ${ }^{15}$ El carácter adecuado de una idea, entonces, consiste en una cierta forma en que se articulan los elementos cognitivos con que se formula la idea, esto es, una forma tal en que dichos elementos presentan la génesis de lo ideado por la idea. Asimismo, en Hegel cada figura de la conciencia presenta una forma en que se articulan los elementos cognitivos (capacidades y conceptos) y prácticos (deseo y reconocimiento) implicados en el saber.

Esta similitud entre lo que Spinoza entiende por idea adecuada y lo que Hegel entiende por saber que aparece se refleja también en los rendimientos que tiene el criterio interno de la verdad en Spinoza. Pues, en su teoría, la idea adecuada cumple el rol de garantizar la correspondencia entre la idea y lo ideado por ella. Al cumplir este rol, las ideas que son adecuadas permiten a la vez la anticipación de la estructura del mundo. Ahora bien, la anticipación de la estructura del mundo por parte del pensamiento (cuando éste forma nada más que ideas adecuadas) no consiste en una anticipación de una cosa a través de una idea, sino que consiste en una anticipación de la estructura básica del mundo a partir de una serie de ideas adecuadas. ${ }^{16} \mathrm{De}$ un modo similar, en Hegel el nuevo saber determina cuál es la estructura ontológica del nuevo objeto, en la medida en que la forma en que se articulan los elementos cognitivos y prácticos del primero anticipan la estructura ontológica del nuevo objeto. Tomaré como ejemplos los tránsitos de Certeza Sensible a Percepción y de Fuerza y Entendimiento a Autoconciencia.

En el caso de la Certeza Sensible, su pretensión de saber era conocer lo inmediato, sensible y singular. La experiencia que esta figura hace es que solo es posible la referencia a un singular mediante conceptos, los cuales son

\footnotetext{
1957.

15 Para ello, puede verse el artículo de Parkinson 1977; también el libro de Hallett

16 Pues la serie que le interesa a Spinoza no es la serie de las cosas mutables, sino la de las cosas eternas (TIE 100).
} 
algo universal en el sentido de ser algo mediado. El tipo de mediación que aparece aquí es la que está presente ejemplarmente en los conceptos de aquí y ahora. La comprensión de lo que es el ahora y el aquí depende de la comprensión de los ahora y aquí singulares en su referencia y articulación mutua; tal referencia y articulación corresponden al concepto que tenemos de ahora y aquí, y solo mediante ellos podemos referirnos a un ahora y aquí singulares.

Ahora bien, en la Percepción, el nuevo objeto contiene esta misma forma: el objeto es ahora una multiplicidad de elementos sensibles singulares, los cuales solo son concebidos en su mutua referencia y articulación, la cual da lugar a lo que Hegel llama la cosa. Así, la cosa es al concepto de aquí y ahora como las propiedades lo son respecto a los aquí y ahora singulares. Pero no solo eso, sino que las propiedades mismas poseen la estructura de la mediación: las propiedades son tales solo en mutua referencia a otras instancias de la misma propiedad y en su diferencia con las otras propiedades. De este modo, el nuevo objeto posee la misma estructura que corresponde a la forma en que se articulan las capacidades cognitivas humanas (en este caso, sensibilidad y formación de conceptos), en la pretensión de saber que es condición de posibilidad del saber examinado (el de la Certeza Sensible).

En el caso de Fuerza y Entendimiento, esta figura hace la experiencia de que lo universal no sensible (el orden legaliforme de la naturaleza) solo puede ser comprendido si el saber tiene la forma de lo infinito: una igualdad que se produce y mantiene por su constante diferenciación y negación de su diferencia. Esta es la manera en que se dispone la forma del saber básica de la autoconciencia. La autoconciencia tiene dos objetos, el objeto sensible y ella misma en cuanto determinación del objeto, y ella, mediante un acto, trata de llevar ambos lados a una unidad, unidad que es el conocimiento del objeto; así, esta unidad es la igualdad que se produce por su diferenciación (escisión del objeto para la autoconciencia) y por la negación de esta diferenciación (acto que lleva a la unidad los dos objetos).

Ahora bien, el objeto sensible de la autoconciencia es algo vivo, y su estructura se corresponde con la forma en que se dispone el saber de ese objeto en la autoconciencia. Lo que está vivo participa del movimiento infinito que constituye la vida. La vida se diferencia a sí misma: se escinde en figuras (seres vivos). Cada una de estas figuras subsiste para sí (se mantiene vivo) en la medida en que devora a la otra, pero con esto no hace sino instanciar la unidad que es la vida, en cuanto proceso vital: la vida como unidad elemental se mantiene a sí misma mediante su división en seres vivos y la aniquilación de un ser vivo por otro. Sin embargo, también el ser 
vivo mismo tiene esta estructura de lo infinito: él mismo encuentra a otro distinto de sí y cancela esa diferencia devorando a este otro, llegando así a la unidad que es él mismo en cuanto ente viviente.

En estos casos se ve claramente que el nuevo objeto surge del nuevo saber en el sentido de que la forma en que se articulan las capacidades cognitivas y prácticas de la conciencia se corresponde con la estructura ontológica que va a manifestar el nuevo objeto. ${ }^{17}$ Con esto tenemos ya las respuestas a las preguntas que guían este escrito. Pues, si cada saber que aparece contiene un criterio interno de la verdad distinto (pues es una articulación distinta de los elementos cognitivos y prácticos implicados en el saber), entonces, necesariamente, la emergencia de un nuevo saber determinará la emergencia de un nuevo objeto, en la medida en que la estructura ontológica del nuevo objeto es anticipada por el criterio interno de la verdad contenido en el saber que aparece. Esta sería la razón por la cual la emergencia del nuevo objeto depende de la modificación en el saber.

Además, esta misma correspondencia entre la forma en que se articula el saber y la estructura ontológica del objeto permite explicar por qué el nuevo objeto es cada vez uno distinto. Pues, al modificar el saber, se modifican los elementos en los cuales éste se articula, y por ende se modifica su forma. En correspondencia, al modificarse la forma en que se articulan los elementos y capacidades cognitivas y prácticas en la pretensión de saber, también se modificará la estructura ontológica del objeto que se corresponde con aquella forma del saber. En consecuencia, en la medida en que el saber se modifica para ajustarse al primer objeto, lo que vale como objeto queda eo ipso modificado, de suerte que surge un nuevo objeto que será siempre diferente del anterior, tal como el nuevo saber es diferente del anterior.

La propuesta que acabo de exponer aquí es todavía solo eso: una propuesta. Falta aún un análisis más detallado en torno a la similitud de los planteos de Hegel y Spinoza, y una mayor precisión sobre cómo funcionaría, en concreto, el uso que Hegel haría del criterio interno de la verdad. Lo que aquí esbocé fue tan solo el esqueleto de la idea, esqueleto que por sí solo era suficiente para responder las preguntas que motivaron esta investigación.

Antes de concluir, es oportuno que me defienda contra una posible objeción, a saber, que en la analogía con Spinoza va implícita la asunción de la tesis del doble aspecto o del paralelismo entre el Pensamiento y la

17 Esto es explícito en el tránsito a Percepción: “Como el principio del objeto es lo universal y es, en su simplicidad, algo mediado, el objeto tiene que expresar esto como su naturaleza en él; se muestra así como la cosa con muchas propiedades" (PhG 111, énfasis mío). 
Extensión, y junto con ello el monismo substancial. Con esto, se me objetaría que esta propuesta asume, como punto de partida del análisis de Hegel, demasiado como para considerarse un punto de partida neutro. Ahora bien, ante esto he de notar lo siguiente: Spinoza, en el TIE, llega a la idea del criterio interno de la verdad (o en su formulación: la verdad es norma de sí) por razones metódicas, no metafísicas. Es más, el que la idea adecuada asegure la correspondencia entre la idea y su objeto, no dice nada aún sobre el estatus ontológico del orden de las ideas y el orden de los objetos; no porque haya correspondencia entre ellos significa que ambos sean dos aspectos (atributos) de una misma substancia.

El desarrollo argumental es el siguiente: dado que es imposible no caer en una regresión al infinito si uno supone que es necesario saber que uno sabe antes de saber algo, entonces es forzoso admitir la tesis de que la verdad es norma de sí, esto es, que hay algo en la manera en que percibimos -cuando esta es la correcta- que nos permite distinguirla de las percepciones que llevan a lo falso. De este modo, si uno considera que la verdad es -o al menos implica- la correspondencia entre la idea y su objeto, entonces hay que admitir que, una vez encontrada la norma de la verdad, si uno percibe de acuerdo con aquella norma, entonces la idea resultante ha de ser verdadera, esto es, ha de corresponder con su objeto. Es importante notar que esta correspondencia no aplica a la serie de las cosas mutables, sino tan solo a la serie de las cosas eternas (TIE 100); por tanto, no se sigue de ella que del solo hecho de pensar un objeto particular (por ejemplo, esta mesa) se pueda anticipar sus características (por ejemplo, su color), sino que solo se sigue que, a partir de la norma de la verdad,uno puede anticipar la estructura del mundo.

Por último, es importante destacar en qué medida mi propuesta se diferencia de la interpretación de Pippin. La diferencia radica en que, en mi propuesta, la anticipación de la estructura del mundo por parte del criterio interno de la verdad no refiere necesariamente a las condiciones de posibilidad de la experiencia de objetos, aunque ciertamente mi propuesta es compatible con esa visión. Esta leve diferencia me permite superar los dos puntos débiles señalados en la interpretación de Pippin. Pues, por un lado, asumir que es posible determinar para cada saber que aparece su criterio interno de la verdad, no determina eo ipso qué saber que aparece, no corresponde a lo que sería la ciencia. Por otro lado, mi propuesta permite leer las tres primeras secciones de la $P h G$ sin apelar a condiciones de posibilidad de la experiencia. 


\section{BIBLIOGRAFIA}

Beiser, Frederick. 2005. Hegel. New York: Routledge.

Brandom, Robert. 2019. A Spirit of Trust. A Reading of Hegel's Phenomenology. Cambridge: Harvard University Press.

Dove, Kenley R. 1998. "Hegel's Phenomenological Method". In Jon Stewart (ed.), The Phenomenology of Spirit Reader. Critical and Interpretive Essays. New York: State University of New York Press: 52-75.

Fink, Eugen. 2011. Hegel. Interpretaciones Fenomenológicas de la Fenomenología del Espíritu (Iván Ortega Rodríguez trad.). Barcelona: Herder.

Forster, Michael. 1989. Hegel and Skepticism. Cambridge: Harvard University Press.

Fulda, Hans-Friedrich. 2008. "'Science of the Phenomenology of Spirit': Hegel's program and its implementation". In Michael Quante y Dean Moyar (eds.), Hegel's Phenomenology of Spirit. A Critical Guide. Cambridge: Cambridge University Press: 21-42.

Hallett, H.F. 1957. Benedictus de Spinoza. The Elements of his Philosophy. London: The Athole Press.

Harris, H.S. 1997. Hegel's Ladder. I: The Pilgrimage of Reason. Cambridge: Hackett Publishing Company.

Hegel, G.W.F. Ciencia de la Lógica. Volumen I: La Lógica Objetiva (1812/1813) (Félix Duque trad.). Madrid: Abada, 2011.

. Fenomenología del Espíritu (Antonio Gómez Ramos trad.). Madrid: Abada, 2010.

. Lecciones sobre la Historia de la Filosofía III, traducido por Wenceslao Roces. México: Fondo de Cultura Económica, 1955.

. Werke in Zwanzig Bänden. Band V: Wissenschaft der Logik I. Frankfurt: Suhrkamp Verlag, 1969.

. Werke in Zwanzig Bänden. Band XX: Vorlesungen über die Geschichte der Philosophie III. Frankfurt: Suhrkamp Verlag, 1971.

Houlgate, Stephen. 2013. Hegel's Phenomenology of Spirit. A Reader's Guide. New York: Bloomsbury.

Hyppolite, Jean. Génesis y Estructura de la Fenomenología del Espíritu de Hegel, traducido por Francisco Fernández Buey. Barcelona: Península, 1974.

Jaeschke, Walter. 2010. "La Experiencia de la Conciencia" (Ana Carrasco Conde trad). In Félix Duque (ed.), Hegel: La Odisea del Espíritu. Madrid: Círculo de Bellas Artes: 35-52.

Labarrièrre, Jean-Pierre. 1985. La Fenomenología del Espíritu de Hegel, traducido por Guillermo Hirata. México: Fondo de Cultura Económica.

Parkinson, G.H.R. 1977. “'Truth Is Its Own Standard': Aspects of Spinoza's Theory of Truth". The Southwestern Journal of Philosophy 8-3: 35-55. 
Pinkard, Terry. 1994. Hegel's Phenomenology. The Sociality of Reason. Cambridge: Cambridge University Press.

Pippin, Robert. 1989. Hegel's Idealism. The Satisfaction of Self-Consciousness. Cambridge: Cambridge University Press.

Pöggeler, Otto. 1973. “Die Komposition der Phänomenologie des Geistes”. In Hand Friedrich Fulda, Dieter Henrich (eds), Materialen zu Hegels Phänomenologie des Geistes. Frankfurt: Suhrkamp Verlag: 329-390.

Siep, Ludwig. 2015. El Camino de la Fenomenología del Espíritu (Carlos Emel Rendón trad.). Barcelona: Anthropos.

Spinoza, Baruch. 2000. Ética Demostrada según el Orden Geométrico (Atilano Domínguez trad.). Madrid: Trotta. 1988. Correspondencia (Atilano Domínguez trad.). Madrid: Alianza. . 2008. Tratado de la Reforma del Entendimiento (Luis Placencia \& Boris Eremiev trads.). Buenos Aires: Colihue.

Stern, Robert. 2002. Hegel and the Phenomenology of Spirit. New York: Routledge.

Trisokkas, Ioannis. 2012. Pyrrhonian Scepticism and Hegel's Theory of Judgment. A Treatise on the Possibi1n1lity of Scientific Inquiry. Boston: Brill.

Valls Plana, Ramón. 1994. Del Yo al Nosotros. Barcelona: PPU.

Westphal, Kenneth. 1998. "Hegel's Solution to the Dilemma of the Criterion". In Jon Stewart (ed.), The Phenomenology of Spirit Reader. Critical and Interpretive Essays. New York: State University of New York Press: 76104.

. 2003. Hegel's Epistemology. A Philosophical Introduction to the Phenomenology of Spirit. Cambridge: Hackett Publishing Company. 